\title{
Short Communications \\ Reduction of Influenza and Enterovirus Infection in Taiwan during the COVID-19 Pandemic
}

\author{
Chi-Hsin Sally Chen ${ }^{1}$, Tsun-Jen Cheng ${ }^{2 *}$ \\ ${ }^{1}$ Master of Public Health Program, College of Public Health, National Taiwan University, Taipei 10055, Taiwan \\ ${ }^{2}$ Institute of Environmental and Occupational Health Sciences, College of Public Health, National Taiwan University, \\ Taipei 10055, Taiwan
}

\begin{abstract}
Coronavirus disease 2019 (COVID-19) caused by severe acute respiratory syndrome coronavirus-2 (SARS-CoV-2) is believed to be transmitted through respiratory droplets, aerosols, and contact. During the COVID-19 pandemic, Taiwan has implemented timely and accurate non-pharmaceutical intervention (NPI) measures on a national level (i.e., border control, quarantine and isolation, contact tracing, delay of school opening, and suspension of mass gatherings), and promoted personal hygiene habits to the public (i.e., frequent hand washing with soap, face masks wearing). Consequently, as of July 27, Taiwan has had relatively low number of confirmed cases (458) and deaths (7), and no locally transmitted cases for more than 30 consecutive days. We observed from data collected via Taiwan National Infectious Disease Statistics System that during the COVID-19 pandemic, outpatient visits for other infectious diseases such as influenza, which is also transmitted through droplets, aerosols, and contact, and enterovirus infections, transmitted via faecal-oral route or respiratory transmissions, have reduced significantly compared to previous two years. Epidemic trend for both diseases have also changed, with seasonal influenza activities ending weeks earlier than it did in 2018 and 2019, and lack of increase in enterovirus infections that is usually witnessed at the end of March. Our observation suggest the NPI measures and personal hygiene habits enforced against the COVID-19 pandemic may have inadvertently "flattened the curve" for influenza and enterovirus infections as well. This may indirectly relieve the stress on Taiwan's health system and healthcare workers during the COVID-19 crisis. More information will be needed to verify this hypothesis.
\end{abstract}

Keywords: COVID-19; Taiwan; Influenza; Enterovirus Infection; Non-pharmaceutical interventions.

In late December 2019, coronavirus 2019 (COVID-19), an infectious disease caused by severe acute respiratory syndrome coronavirus-2 (SARS-CoV-2), broke out in Wuhan City, China, and has now become a global crisis. As of July 27 , more than 16 million confirmed cases and 600 thousand deaths have been reported worldwide (John Hopkins University, 2020). COVID-19 is believed to be transmitted through respiratory droplets $(5-10 \mu \mathrm{m})$. However, studies have shown plausible aerosol $(<5 \mu \mathrm{m})$ transmission of the virus as well, and viable virus has been detected on surfaces such as plastic for up to 72 hours (van Doremalen et al., 2020). SARS-CoV-2 vRNA was also detected in air samples collected at a healthcare facility, along with other respiratory viruses including Influenza A H1N1 and Influenza A H3N2 (Lednicky et al., 2020). There is currently no specific treatment or

\footnotetext{
* Corresponding author.

Tel.: +886-2-3366-8090

E-mail address: tcheng@ntu.edu.tw
}

vaccine for COVID-19. Although experimental treatments have been given in countries amidst the worst COVID-19 outbreaks, non-pharmaceutical interventions (NPIs) have been the primary measure for disease outbreak control (WHO, 2019). Compared to other countries, Taiwan has so far been successful in controlling the outbreak, with 458 confirmed cases, 7 deaths, and no locally transmitted cases for more than 30 days (as of July 27) (TCDC, 2020a). Aside from NPIs implemented at a national level (i.e., border control, isolation and quarantine, contact tracing, delay of school, suspend mass gatherings), Taiwan Centers for Disease Control (TCDC) also issued guidelines for personal prevention including practice of good hygiene habits such as frequent hand washing with soap, refrain from face touching, cough etiquette, and use of masks (TCDC, 2020b, c). Timely and accurate implementation of these NPIs have allowed Taiwan to contain the epidemic without resorting to strict lockdowns that restrict freedom of movement, disrupt everyday life, and have a devastating effect on the economy.

Personal preventative steps for COVID-19 are similar to those advised for other respiratory infections such as 
influenza. Influenza is an acute respiratory disease caused by influenza $\mathrm{A}, \mathrm{B}$, and $\mathrm{C}$ viruses, which binds to receptors in both upper and lower respiratory tracts, and is transmitted like COVID-19 through aerosal, droplets, and contact (Paules and Subbarao, 2017). Frequent hand washing and social distancing are also recommended for other contact transmitted infectious diseases such as enterovirus infections. Enterovirus are small RNA viruses including polioviruses, coxsackie viruses, echoviruses, and numbered enteroviruses such as EVA71. These viruses are found in the gastrointestinal or respiratory tract where they primarily replicate, and could spread either via faecal-oral route or respiratory transmissions by droplets and aerosols (Baggen et al., 2018). Previous studies have found significantly higher positive rate for enterovirus from throat swabs $(90 \%)$ compared to rectal swabs $(32 \%)$, and airborne routes of transmission for enterovirus have also been reported (Chang et al., 1999; Couch et al., 1970; Gonzalez-Martin et al., 2018). On this basis, we collected data from Taiwan National Infectious Disease Statistics System, an open online database for updated information on notifiable infectious disease in Taiwan, to compare trends of infectious diseases in 2020 to the same period in 2018 and 2019, and observed significant changes for influenza and enterovirus infection (TCDC, 2020d).

Seasonal influenza epidemics are caused by influenza A and $\mathrm{B}$ viruses that routinely spread in a human population. In Taiwan, flu season typically begins from December, and peaks during the Lunar New Year holiday which is followed by the beginning of school spring semester, around January to February of the following year (Su et al., 2019). Seasonal influenza still poses a major public health threat in Taiwan, accounting for 114 and 82 deaths in the 2017-2018 and 2018-2019 flu season, respectively (TCDC, 2018, 2019). For enteroviruses, EVA71 infections are usually associated with severe complications, while infection with other nonpolio enteroviruses present milder symptoms. Annually in Taiwan, enterovirus infections start to increase in late March, and reach a peak around mid-June. The epidemic curve then decreases gradually, usually with another outbreak of a smaller scale in September when school begins. Coxsackie A viruses are the major cause of annual outbreaks, while an EVA71 epidemic occurs every 3-4 years (Liu et al., 2016). There are currently no non-polio vaccines in Taiwan, and children under five years of age are most susceptible to infections and severe complications.

According to TCDC reports, in week 1 of 2020, seasonal influenza epidemic was on the rise with increasing medical visits, and a total of 499 cases with severe complications, 26 being fatal, since the season began at the end of 2019 (TCDC, 2020e). As shown in Fig. 1, based on data we acquired from Taiwan National Infectious Disease Statistics System, 71609 outpatient visits were recorded during this week, which was significantly higher than week 1 of 2018 (58827) and 2019 (40649). However, the numbers began to drop after week 2 in 2020, with a sharp decline seen between weeks 4 and 5 (64807 and 43513 outpatient visits, respectively) when the Lunar New Year started and influenza cases were expected peak as it did in 2018 (weeks 7 and 8, with 59542 and 69101 outpatient visits, respectively) and 2019 (weeks 6 and 7, with 27445 and 56676 outpatient visits, respectively). By week 9, outpatient visits had dropped below 20000, and TCDC announced seasonal influenza activity had ended, weeks earlier than it was declared in 2018 (week 15) and 2019 (week 12). The most updated data we have access to is for week 19 of 2020, with 6324 outpatient visits, an 11-fold decrease from week 1 , and significantly lower than the numbers reported for the same week in 2018 (16511) and 2019 (31785). Fig. 2 shows that based on the data we collected

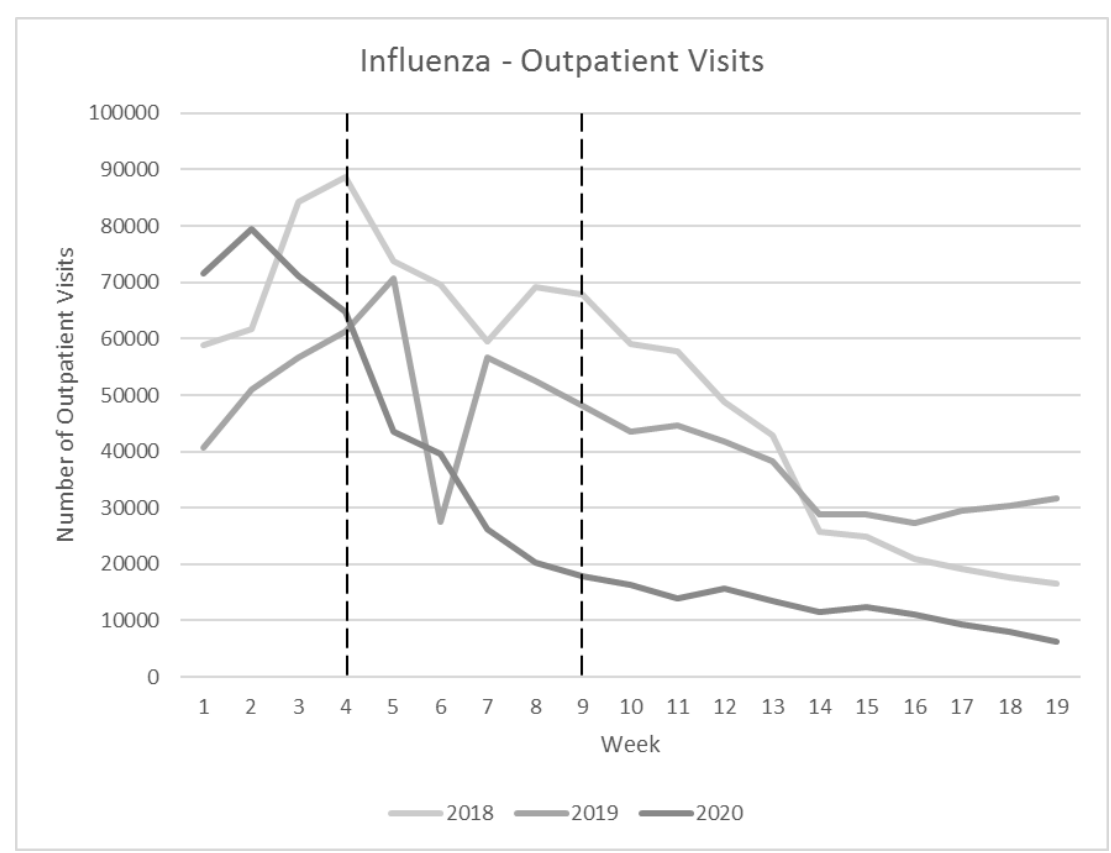

Fig. 1. Weekly number of outpatient visits for influenza in Taiwan during week 1 to 19 from 2018 to 2020 . (Week 4: implementation of face mask policies; Week 9: delayed start of school spring semester) 


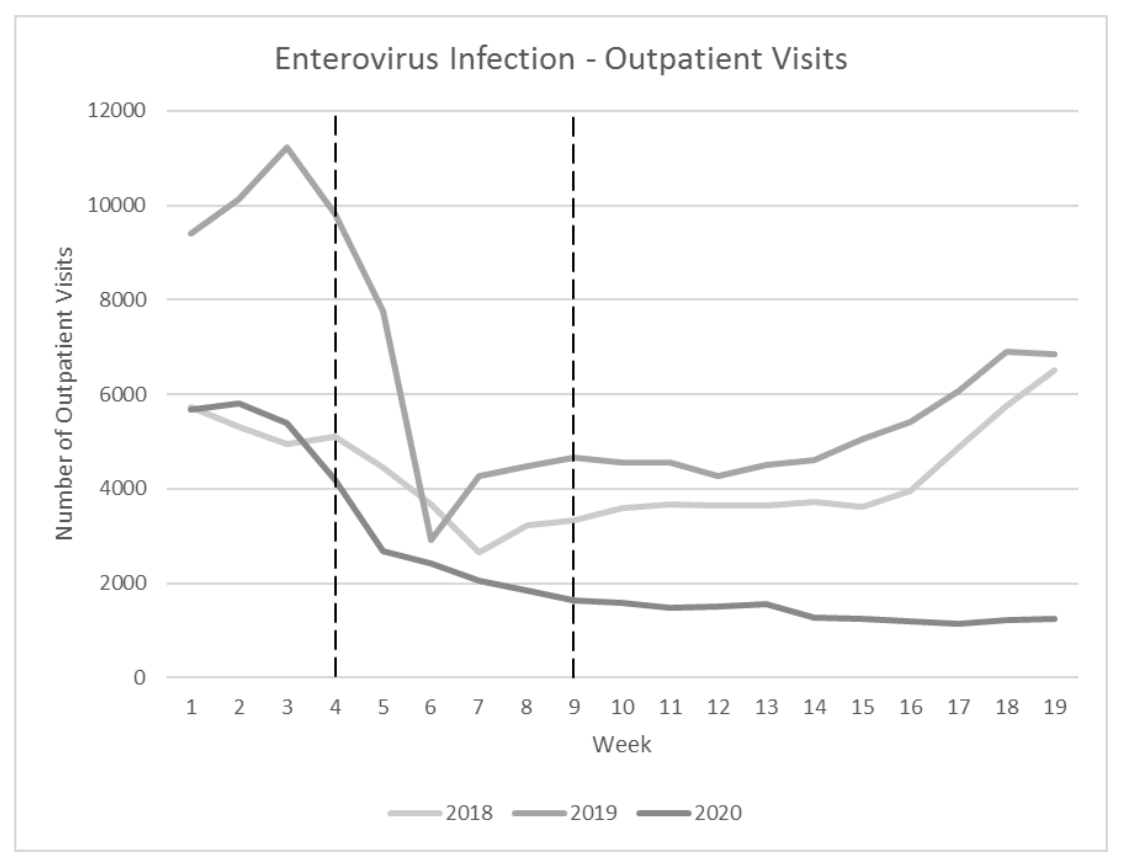

Fig. 2. Weekly number of outpatient visits for enteroviral infection in Taiwan during week 1 to 19 from 2018 to 2020. (Week 4: implementation of face mask policies; Week 9: delayed start of school spring semester)

from Taiwan National Infectious Disease Statistics System, enterovirus infections did not start out so high at the beginning (week 1) of 2020 with 5678 outpatient visits compared to 5719 in 2018 and 9397 in 2019 . However, the small increase in week 7 of 2018 and week 6 of 2019 that coincided with Lunar New Year and start of school was avoided this year, and the number of outpatient visits declined steadily until week 19 (1246), with no sign of increase at the end of March (week 14) as it did in 2018 and 2019.

In order to address whether the decrease in outpatient visits for influenza and enterovirus were also influenced by the population avoiding clinics/hospitals due to COVID-19 fears, we looked at the data on severe complicated influenza and enterovirus infections also provided on Taiwan National Infectious Disease Statistics System (data not shown). The number of cases for severe complicated influenza in week 1 of 2020 was 100 , more than two times higher than it was in 2019 (44 confirmed cases), but the numbers dropped dramatically to 0 cases in week 9 of 2020 and have remained low with reports of one confirmed case each in weeks 11 , 21 , and 26. In week 9 of 2019, the number of confirmed cases was still at 53, and throughout 2019 never dropped below 17. We could also rule out the contribution of imported cases, since there were only 4 import cases in 2019, and 2 in 2020. For enterovirus infection with severe complications, the data available so far (week 1-31) showed there have been only 5 confirmed cases in 2020, which is significantly lower compared to the same period in 2019 (29) and 2018 (26). There have also been no confirmed cases since week 8 in 2020 .

The steady decline we observed in both influenza and enterovirus infections may be an inadvertent result of Taiwan government's implementation of NPIs and promotion of good personal hygiene habits in the population for COVID-19 control. Under this hypothesis, the NPIs (e.g., suspension of mass gatherings) and habits of the general public (e.g., mask wearing) reduced the aerosol, droplet, and contact transmission of these other viruses, resulting in a cobenefit of the COVID-19 prevention measures. For instance, suspension of mass gatherings as a social distancing measure reduced the probability of aerosol and droplet transmission not just of COVID-19 but influenza and enteroviruses as well, while mask-wearing directly blocked a fraction of these aerosols and droplets, further reducing the transmission probability. Central Epidemic Command Center (CECC) was established on January 20 (week 4), and the next day Taiwan had the first confirmed case of COVID-19. January 22, CECC began to hold daily press conferences informing the public of the epidemic progress, and governmental guidelines and policies. On January 24, export of N95 and surgical masks were restricted and on January 30 (week 5) nation-wide acquisition of domestically produced surgical masks was announced. Name-based face mask rationing system began on February 6 (week 6). All of these measures, combined with people's experience during the 2003 SARS outbreak, potentially raised people's awareness of self-protection by wearing face masks in public before it was mandated at the end of March. Taiwan was also one of the first countries to announce a delayed school opening this year (February 2-3, week 5), which allowed Ministry of Education and schools more time to adopt necessary precaution measures, including distribution of face masks, forehead thermometers, and alcohol disinfectant. Local environment protection bureaus also helped to disinfect public facilities in 4000 senior high schools and under nationwide before schools started on February 25 (week 9). As a result, Taiwan is now one of the few countries in the world where students are still able to attend school in person.

From our observation, Taiwan government and society's 
joint efforts in containing and mitigating the COVID-19 pandemic may have unintentionally "flattened the curve" for influenza and enterovirus infections as well. This could indirectly help preserve the health system capacity and reduce stress for healthcare workers. In the future, we will continue using the Taiwan National Infectious Disease Statistics System to follow up on the influenza and enterovirus infection situation in Taiwan in order to verify our hypothesis as the COVID-19 pandemic progresses.

\section{REFERENCES}

Baggen, J., Thibaut, H.J., Strating, J. and van Kuppeveld, F.J.M. (2018). The life cycle of non-polio enteroviruses and how to target it. Nat. Rev. Microbiol. 16: 368-381. https://doi.org/10.1038/s41579-018-0005-4

Chang, L.Y., Lin, T.Y., Huang, Y.C., Tsao, K.C., Shih, S.R., Kuo, M.L., Ning, H.C., Chung, P.W. and Kang, C.M. (1999). Comparison of enterovirus 71 and coxsackievirus a16 clinical illnesses during the taiwan enterovirus epidemic, 1998. Pediatr. Infect. Dis. J. 18: 1092-1096.

Couch, R.B., Douglas, R.G.J., Lindgren, K.M., Gerone, P.J. and Knight, V. (1970). Airborne transmission of respiratory infection with coxsackievirus a type 2112 . Am. J. Epidemiol. 91: 78-86. https://doi.org/10.1093/oxf ordjournals.aje.a121115

Gonzalez-Martin, C., Coronado-Alvarez, N.M., TeigellPerez, N., Diaz-Solano, R., Exposito, F.J., Diaz, J.P., Griffin, D.W. and Valladares, B. (2018). Analysis of the impact of african dust storms on the presence of enteric viruses in the atmosphere in Tenerife, Spain. Aerosol Air Qual Res 18: 1863-1873. https://doi.org/10.4209/aaqr.20 17.11.0463

John Hopkins University (2020). COVID-19 dashboard. https://coronavirus.jhu.edu/map.html

Lednicky, J.A., Shankar, S.N., Elbadry, M.A., Gibson, J.C., Alam, M.M., Stephenson, C.J., Eiguren-Fernandez, A., Morris, J.G., Mavian, C.N., Salemi, M., Clugston, J.R. and Wu, C.Y. (2020). Collection of SARS-CoV-2 virus from the air of a clinic within a university student health care center and analyses of the viral genomic sequence. Aerosol Air Qual Res 20: 1167-1171. https://doi.org/10.4 209/aaqr.2020.05.0202

Liu, D.P., Wang, T.A., Huang, W.T., Chang, L.Y., Wang, E.T., Cheng, S.H. and Yang, M.C. (2016). Disease burden of enterovirus infection in Taiwan: Implications for vaccination policy. Vaccine 34: 974-980. https://doi.org/ 10.1016/j.vaccine.2015.12.026

Paules, C. and Subbarao, K. (2017). Influenza. Lancet 390:
697-708. https://doi.org/10.1016/S0140-6736(17)30129-0 Su, C.P., Tsou, T.P., Chen, C.H., Lin, T.Y. and Chang, S.C. (2019). Seasonal influenza prevention and control in Taiwan-Strategies revisited. J. Formos. Med. Assoc. 118: 657-663. https://doi.org/10.1016/j.jfma.2018.12.022

Taiwan Centers for Disease Control (TCDC) (2018). Taiwan influenza express week 15 2017-2018 influenza season. https://www.cdc.gov.tw/En/File/Get/5RgNeUAZ uQ4hkHWFPNrr7Q

Taiwan Centers for Disease Control (TCDC) (2019). Taiwan influenza express week 12 2018-2019 influenza season. https://www.cdc.gov.tw/En/File/Get/M7be7DFS giRWTfEOjb92_g

Taiwan Centers for Disease Control (TCDC) (2020a). COVID-19 (2019-nCoV). https://sites.google.com/cdc.g ov.tw/2019-ncov/taiwan

Taiwan Centers for Disease Control (TCDC) (2020b). Prevention and control of COVID-19 in Taiwan. htt ps://www.cdc.gov.tw/En/Category/Page/0vq8rsAob_9H Ci5GQ5jH1Q

Taiwan Centers for Disease Control (TCDC) (2020c). Guidelines for prevention of SARS-CoV-2 infection. https://www.cdc.gov.tw/File/Get/HAvRHGs_EjKeROH YmzWm5w

Taiwan Centers for Disease Control (TCDC) (2020d). Taiwan National Infectious Disease Statistics System. http://nidss.cdc.gov.tw/en/

Taiwan Centers for Disease Control (TCDC) (2020e) Taiwan influenza express week 1 2019-2020 influenza season. https://www.cdc.gov.tw/En/File/Get/C1-0p0p_L dXeuhUNb_z2ZA

van Doremalen, N., Bushmaker, T., Morris, D.H., Holbrook, M.G., Gamble, A., Williamson, B.N., Tamin, A., Harcourt, J.L., Thornburg, N.J., Gerber, S.I., Lloyd-Smith, J.O., de Wit, E. and Munster, V.J. (2020). Aerosol and surface stability of SARS-CoV-2 as compared with SARS-CoV-1. N. Engl. J. Med. 382: 1564-1567. https://doi.org/10.1056/ NEJMc2004973

World Health Organization (WHO) (2019). Nonpharmaceutical public health measures for mitigating the risk and impact of epidemic and pandemic influenza. Annex: Report of systematic literature reviews. World Health Organization, Geneva, Switzerland.

Received for review, May 20, 2020 Revised, July 31, 2020 Accepted, August 12, 2020 